\title{
Abundance Ratios in Open Clusters and Field Giants of the Outer Galactic Disk
}

\author{
David Yong \& Bruce W. Carney \\ Department of Physics \& Astronomy, University of North Carolina, Chapel Hill, NC \\ 27599-3255, USA \\ email: yong,bruce@physics.unc.edu
}

\begin{abstract}
We present elemental abundance ratios for bright red giants in the old, distant open clusters Berkeley 20, Berkeley 21, NGC 2141, Berkeley 29, and Berkeley 31 as well as for distant field red giants in the direction of the southern Galactic warp. At Galactocentric distances beyond 10 to $12 \mathrm{kpc}$, the metallicity gradient disappears. The open cluster and field red giants of the outer disk exhibit an almost constant iron abundance $[\mathrm{Fe} / \mathrm{H}]=-0.5$. We also measured the abundances for 15 other elements and found very similar compositions in the field and cluster stars. Specifically, they all show enhancements of the $\alpha$ elements $\mathrm{O}, \mathrm{Mg}, \mathrm{Si}, \mathrm{Ca}$, and $\mathrm{Ti}$ as well as the r-process element $\mathrm{Eu}$. These abundance patterns reveal a rapid star formation history for the outer Galactic disk. A possible explanation for the measured compositions is that the outer disk formed as a result of star formation triggered by a series of merger or accretion events.
\end{abstract}

Keywords. Open clusters and associations: general, stars: abundances, Galaxy: abundances, Galaxy: disk

\section{Target Selection, Observations, and Analysis}

Our goal was to measure metallicities $[\mathrm{Fe} / \mathrm{H}]$ and elemental abundance ratios $[\mathrm{X} / \mathrm{Fe}]$ for old open cluster giants as well as field giants in the outer Galactic disk. The open cluster candidates were selected from optical and infrared color-magnitude diagrams. Using the echelle spectrographs on the $4 \mathrm{~m}$ telescopes at KPNO and CTIO, radial velocities of open cluster candidates were measured from short exposures. Longer exposures of radial velocity members were then obtained in order to conduct the abundance analysis. Candidate field giants were selected from the VI photometry by Carney \& Seitzer (1993). Radial velocities were measured using the Argus multi-object spectrograph at CTIO. Stars with large radial velocities were then observed using the CTIO echelle spectrograph.

Initial estimates for the stellar parameters were based on photometry. Spectroscopic constraints (i.e., excitation and ionization equilibrium) were used to set the final values. For field and cluster giants, elemental abundance ratios were measured for various $\alpha$, iron-peak, and neutron-capture elements using LTE model atmospheres computed using ATLAS9 (Kurucz 1993) and an updated version of the LTE stellar line analysis program MOOG (Sneden 1973).

\section{Results and Conclusions}

We confirm the lack of an age-metallicity relation for the outer disk open clusters. For Be 21, our observed star is rapidly rotating and Li-rich, the second Li-rich star found in this cluster. For both the field and cluster giants, $[\mathrm{Fe} / \mathrm{H}]$ appears to reach a basement value of $[\mathrm{Fe} / \mathrm{H}]=-0.5$ beyond $R_{\mathrm{GC}}=10 \mathrm{kpc}$ in agreement with the predictions by Chiappini et al. (2001). The outer disk open cluster and field giants all show enhancements for $[\alpha / \mathrm{Fe}]$, the $r$-process element $\mathrm{Eu}$, and the $s$-process elements. 


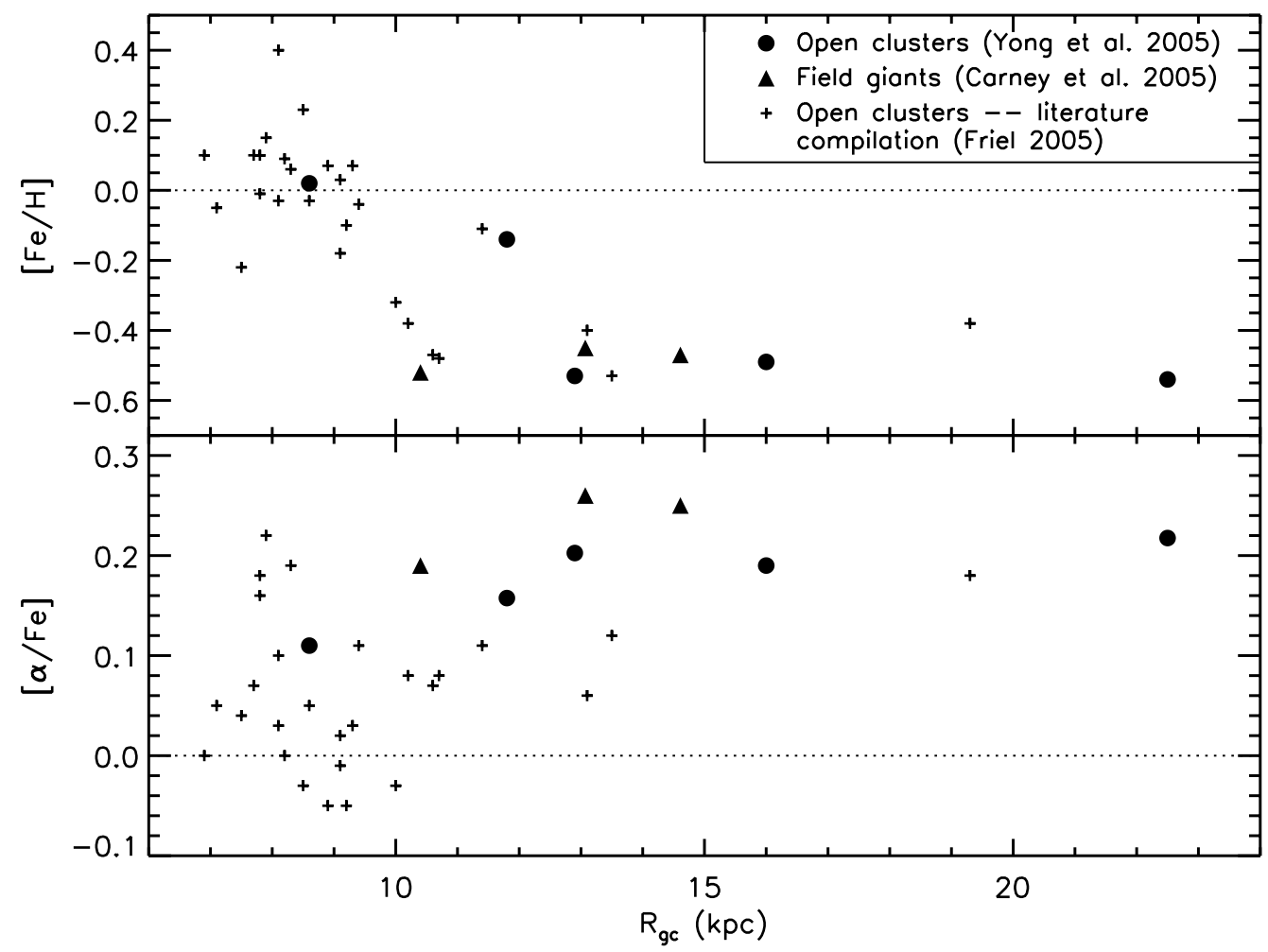

Figure 1. $[\mathrm{Fe} / \mathrm{H}]$ versus Galactocentric distance (upper) and $[\alpha / \mathrm{Fe}]$ versus Galactocentric distance (lower).

The compositions, kinematics, and ages of the outer disk field and cluster stars do not match any known stellar population (thin disk, thick disk, halo, bulge, dwarf spheroidal galaxies). The enhanced $[\alpha / \mathrm{Fe}]$ indicates a rapid star formation history for the outer disk. A series of merger or accretion events likely played a prominent role in the formation and evolution of the outer disk. Our preliminary analysis of distant Cepheids also reveals unusual abundance trends for the outer Galactic disk (see contribution by Carney \& Yong (2005) in this volume).

\section{Acknowledgements}

This research has been supported by the US National Science Foundation through grant AST 03-05431 to the University of North Carolina.

\section{References}

Carney, B.W. \& Seitzer, P. 1993, AJ, 105, 2127

Carney, B.W. Yong, D., de Almeida, M. L. T., \& Seitzer, P. 2005 AJ in press

Carney, B.W. \& Yong, D. 2005 IAU Symposium 228

Chiappini, C., Matteucci, F., \& Romano, D. 2001, ApJ, 554, 1044

Friel, E.D. 2005, private communication

Kurucz, R. 1993, ATLAS9 Stellar Atmosphere Programs. Kurucz CD-ROM No. 13.

Sneden, C. 1973, ApJ, 184, 839

Yong, D., Carney, B.W., \& de Almeida, M.L.T. 2005, AJ in press 


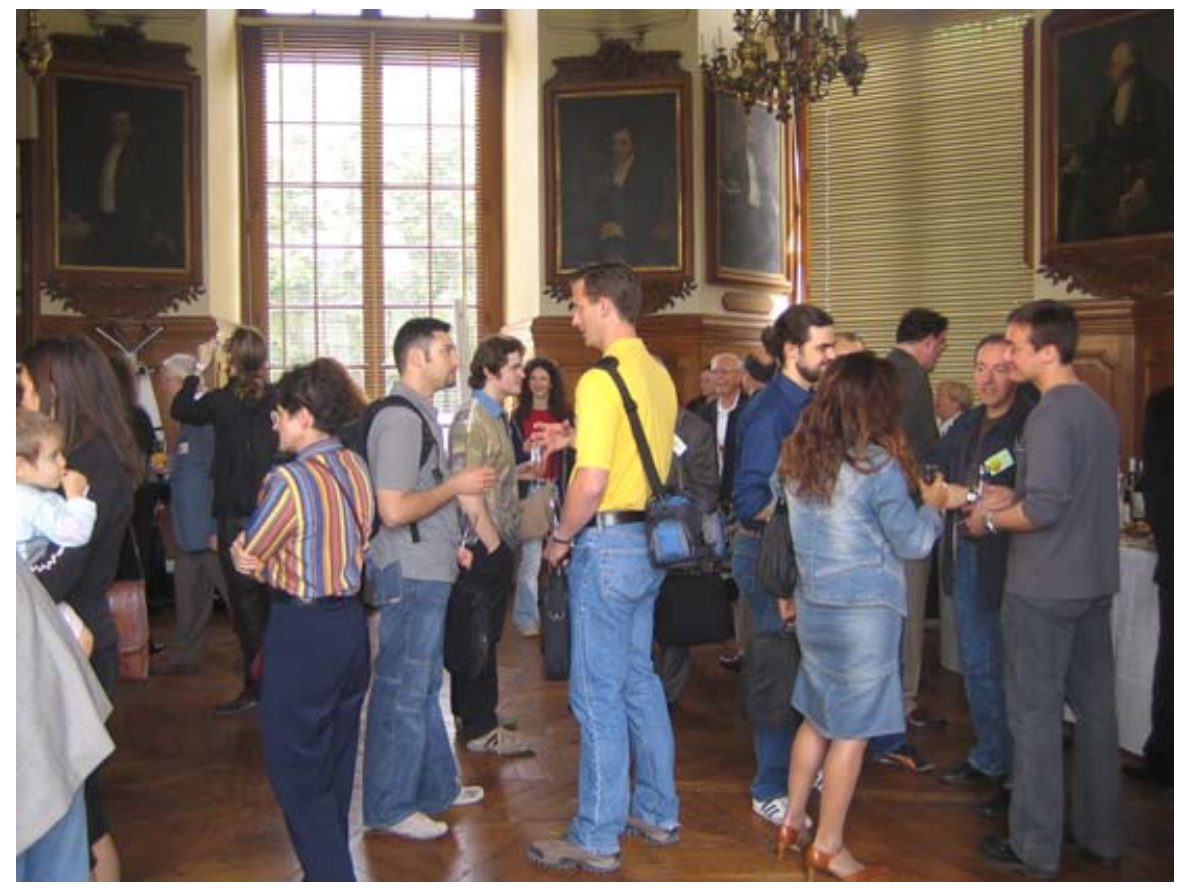

Thomas Bensby, among many other participants, at the welcome reception.

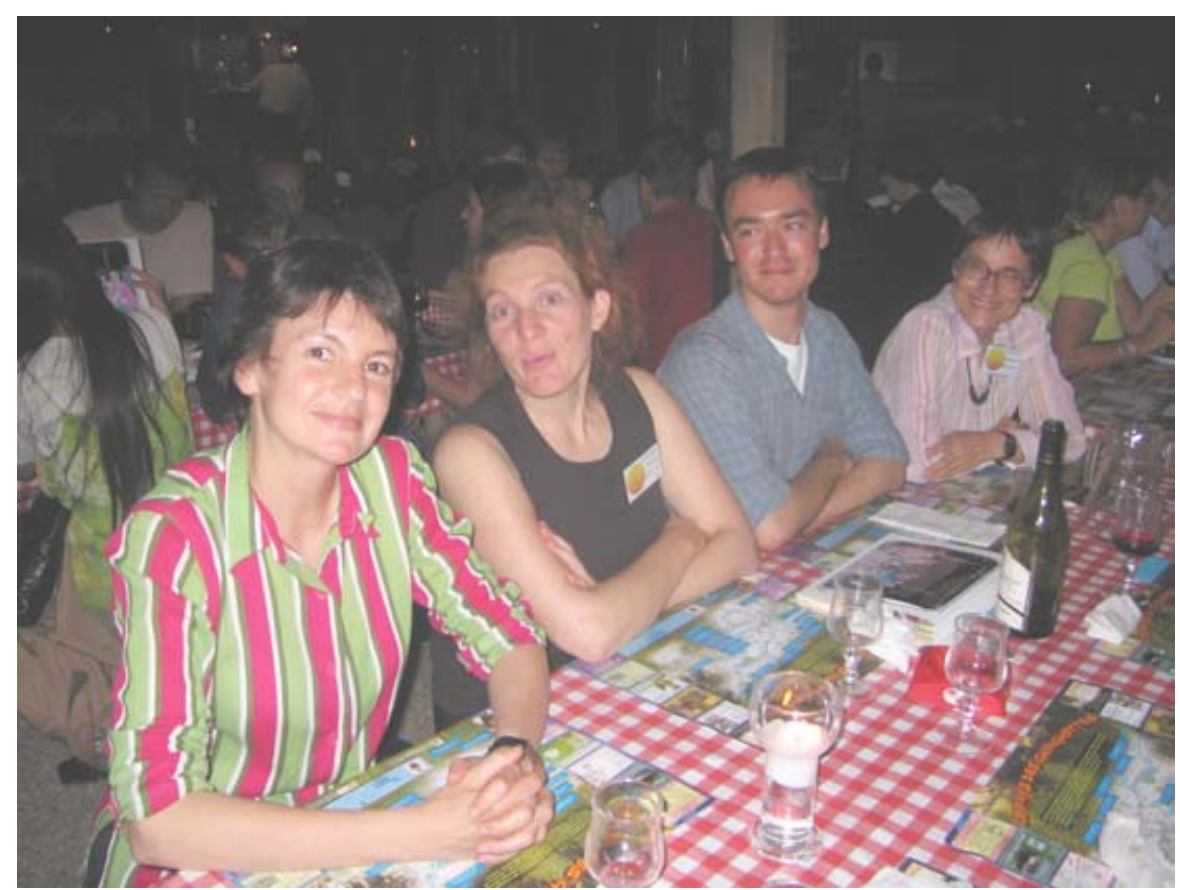

Part of the conference LOC (Pascale Jablonka, Vanessa Hill and Gaël James) alongside Monique Spite at the conference dinner. 


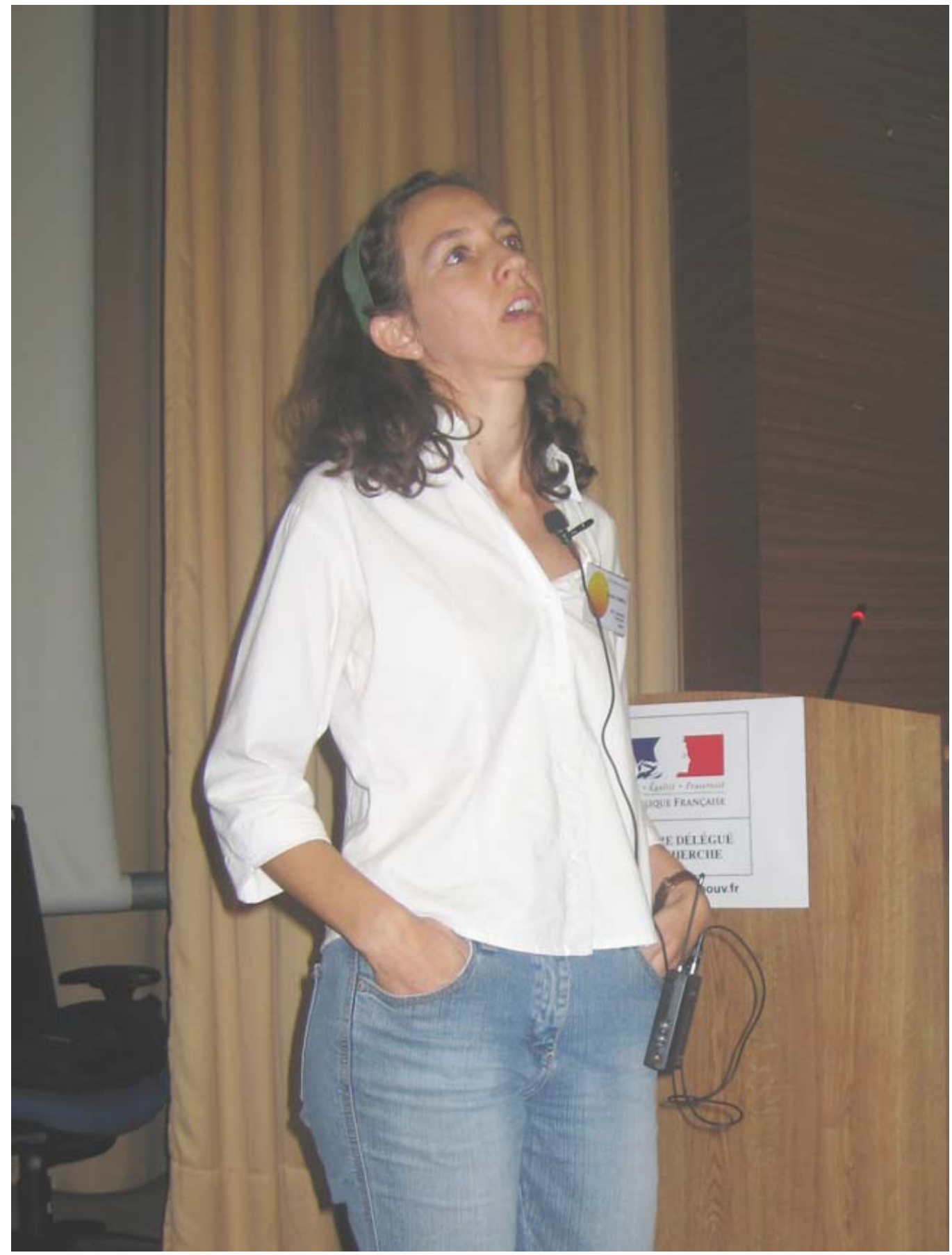

Luciana Pompeia answering questions about the LMC chemical evolution. 